\title{
Anti-oncogenic activities of cyclin D1b siRNA on human bladder cancer cells via induction of apoptosis and suppression of cancer cell stemness and invasiveness
}

\author{
CHUL JANG KIM ${ }^{1}$, TOKIO TERADO ${ }^{2}$, YUKIHIRO TAMBE ${ }^{3}$, KEN-ICHI MUKAISHO ${ }^{4}$, \\ HIROYUKI SUGIHARA ${ }^{4}$, AKIHIRO KAWAUCHI ${ }^{5}$ and HIROKAZU INOUE ${ }^{3}$ \\ ${ }^{1}$ Department of Urology, Kohka Public Hospital, Minakuchi-cho, Kohka, Shiga 528-0074; \\ ${ }^{2}$ Department of Stem Cell Biology and Regenerative Medicine, ${ }^{3}$ Division of Microbiology and \\ Infectious Diseases, ${ }^{4}$ Division of Molecular and Diagnostic Pathology, ${ }^{5}$ Division of Urology, \\ Shiga University of Medical Science, Setatsukinowa-cho, Otsu, Shiga 520-2192, Japan
}

Received August 28, 2017; Accepted October 30, 2017

DOI: $10.3892 /$ ijo.2017.4194

\begin{abstract}
The human cyclin Dl gene generates two major isoforms, cyclin Dla and cyclin Dlb, by alternative splicing. Although cyclin Dlb mRNA is hardly expressed in normal human tissues, it is detected in approximately $60 \%$ of human bladder cancer tissues and cell lines. In the present study, to assess the therapeutic ability of cyclin DIb siRNA, we investigated the anti-oncogenic effects of cyclin DIb siRNA on human bladder cancer cell lines, SBT31A and T24, which express cyclin Dlb mRNA. Knockdown of cyclin Dlb by specific siRNA significantly suppressed cell proliferation, in vitro cell invasiveness and three-dimensional (3D) spheroid formation in these cell lines. Cell cycle analyses revealed that cyclin DIb siRNA inhibited G1-S transition in T24 cells. The increase in the sub-G1 fraction, morphological aberrant nuclei with nuclear fragmentation and caspase- 3 activity in SBA31A cells treated with $c y c l i n$ Dlb siRNA showed that $c y c l i n$ Dlb siRNA induced apoptosis. In T24 cells, knockdown of cyclin Dlb suppressed the expression of the stem cell marker CD44. Knockdown of cyclin Dlb or CD44 suppressed the invasiveness under 3D spheroid culture conditions and expression of $\mathrm{N}$-cadherin. Tumor growth of SBT31A cells in nude mice was significantly inhibited by cyclin DIb siRNA. Taken together, these results indicate that knockdown of cyclin Dlb suppresses the malignant phenotypes of human bladder cancer cells via induction of apoptosis and suppression of cancer cell stemness and epithelial-mesenchymal transition. Applying cyclin Dlb
\end{abstract}

Correspondence to: Dr Hirokazu Inoue, Division of Urology, Shiga University of Medical Science, Setatsukinowa-cho, Otsu, Shiga 520-2192, Japan

E-mail: hirokazu@belle.shiga-med.ac.jp

Key words: cyclin D1b, siRNA, bladder cancer, apoptosis, cancer stem cell
siRNA will be a novel therapy for cyclin $D 1 b$-expressing bladder cancers.

\section{Introduction}

Human cyclin Dl generates two major isoforms via alternative splicing: cyclin Dla and cyclin Dlb. The cyclin Dla mRNA, which contains five exons, is $4.5 \mathrm{~kb}$ in length and has a coding region of $882 \mathrm{bp}$. The $1.7 \mathrm{~kb}$ cyclin Dlb mRNA lacks exon 5, but has an extended exon 4 because of non-splicing, i.e., it extends into intron 4 , which contains a translation stop codon after $99 \mathrm{bp}$ and a polyadenylation signal $450 \mathrm{bp}$ downstream of this stop codon. This mRNA encodes a 275 amino acid protein that differs at the $C$ terminus from the 294 amino acid protein encoded by the cyclin Dla mRNA (1). Cyclin Dla is known to be expressed ubiquitously and to regulate the G1-S transition of the cell cycle by binding cyclin-dependent kinase (CDK) 4/6 to the phosphorylated $\mathrm{Rb}$ protein $(2,3)$. Although cyclin Dlb expression has hardly been detected in normal tissues, it has been detected in certain types of cancers, including Ewing's sarcoma, mantle cell lymphoma, bladder, esophageal and colon cancers, B-lymphoid malignancies and breast and prostate cancers (4). Transfection experiments have shown that cyclin Dlb is more oncogenic than cyclin Dla (5-8). Despite the importance of cyclin Dlb in human cancers, few studies have assessed the factors that influence its production or the relative expression of each of its isoforms (9-12). We have shown that ectopic cyclin Dlb expression promoted cell invasiveness and anchorage-independent growth in bladder cancer cells (13). To clarify the oncogenic potential of the cyclin Dlb variant, we previously developed cyclin Dlb transgenic (Tg) mice and found that rectal tumors had developed in $62.5 \%$ $(15 / 24)$ of female $\mathrm{Tg}$ mice, demonstrating the in vivo function of cyclin Dlb as an oncogene (14). We established a cell line, DlbTgRT, derived from a rectal cancer of female cyclin Dlb Tg mouse (14). Small interfering (si) RNA-induced cyclin Dlb knockdown in this cell line suppressed anchorage-independent growth, cell invasiveness and tumorigenicity in nude mice, indicating that cyclin Dlb contributes to rectal carcinogenesis 
and that knockdown of cyclin Dlb is useful for the suppression of malignant phenotypes of cancer cells expressing cyclin D1b. We also found that $c y c l i n$ Dlb activates Erk via activation of Akt and that enhanced phosphorylation of Akt by cyclin Dlb contributes to tumorigenicity (15). In the present study, we investigated the role of cyclin Dlb on malignant phenotypes of human bladder cancer cell lines, T24 and SBT31A, using cyclin DIb siRNA and found that knockdown of cyclin DIb was able to induce apoptosis and suppress stemness and invasiveness of these cells. These findings suggest that knockdown of cyclin Dlb using siRNA is available as a novel therapeutic tool for the treatment of human bladder cancers.

\section{Materials and methods}

Cell culture. Human bladder cancer cell lines, SBT31A (16) and T24, were cultured using RPMI-1640 medium supplemented with $10 \%$ fetal calf serum (FCS), penicillin $(100 \mathrm{U} / \mathrm{ml})$ and streptomycin $(100 \mu \mathrm{g} / \mathrm{ml})$ at $37^{\circ} \mathrm{C}$ in a humidified $5 \% \mathrm{CO}_{2}$ atmosphere. SBT31A was established in our laboratory (16) and maintained from 1998. T24 was purchased from the American Type Culture Collection (ATCC; Manassas, VA, USA) in 2000 and maintained in our laboratory. T24 and SBT31A were authenticated by short tandem repeat analyses.

Cell proliferation assay. Cell proliferation was examined by culturing the cells in $35-\mathrm{mm}$ culture dishes. In brief, $2 \times 10^{4}$ cells were inoculated in triplicate into each dish containing RPMI-1640 medium supplemented with $10 \%$ FCS and incubated at $37^{\circ} \mathrm{C}$ in a humidified $5 \% \mathrm{CO}_{2}$ atmosphere. Viable cells were counted using a hemocytometer.

Fluorescence-activated cell sorting (FACS) analysis. Cell cycle distribution was detected using the Cycletest Plus DNA Reagent kit (Becton-Dickinson, Franklin Lakes, NJ, USA) with a FACSCanto II flow cytometer system and ModFit 3.2 (Becton-Dickinson). In brief, the cells were collected after treatment with cyclin DIb siRNA and fixed in $70 \%$ ethanol at $-20^{\circ} \mathrm{C}$ for $18 \mathrm{~h}$. The cells were washed twice with phosphatebuffered saline (PBS) and then pelleted by centrifugation at $400 \mathrm{x}$ g. The cell pellets were incubated with $250 \mu \mathrm{l}$ of solution A (trypsin in a spermine tetrahydrochloride detergent buffer) at room temperature for $10 \mathrm{~min}, 200 \mu \mathrm{l}$ of solution $\mathrm{B}$ (trypsin inhibitor and ribonuclease $\mathrm{A}$ in citrate stabilizing buffer with spermine tetrahydrochloride) at room temperature for $10 \mathrm{~min}$, and $200 \mu \mathrm{l}$ of solution $\mathrm{C}$ (propidium iodide and spermine tetrahydrochloride in citrate stabilizing buffer) in the dark at $4^{\circ} \mathrm{C}$ for $10 \mathrm{~min}$. Cells were then analyzed by FACSCanto II flow cytometry.

In vitro invasion assay. The in vitro invasive potential of cancer cells was determined using a Matrigel $^{\mathrm{TM}}$ Basement Membrane Matrix Invasion Chamber (chamber size, $6.4 \mathrm{~mm}$; membrane surface area, $0.3 \mathrm{~cm}^{2}$; pore size, $8 \mu \mathrm{m}$; BD Biosciences, Bedford, MA, USA), according to the manufacturer's instructions (13). We added $500 \mu \mathrm{l}$ of cell suspension (5x10 4 SBT31A and $2 \times 10^{4} \mathrm{~T} 24$ cells $\left./ \mathrm{ml}\right)$ to each chamber. The chambers containing the SBT31A and T24 cells were incubated for 3 and 2 days in a humidified $5 \% \mathrm{CO}_{2}$ atmosphere, respectively. Non-invasive cells were removed from the upper surface of the membrane using a cotton swab. The invasive cells on the underside of the membrane were stained using Diff-Quik ${ }^{\mathrm{TM}}$ (Kokusai Shiyaku Co., Ltd., Kobe, Japan) and counted under a microscope. Each sample was then assayed in triplicate.

Reverse transcriptase-polymerase chain reaction (RT-PCR) analysis. Total RNA was isolated from cultured cells using TRIzol reagent (Invitrogen, Carlsbad, CA, USA). Isolated RNA was used for First-Strand cDNA Synthesis using SuperScript II (Invitrogen) and an oligo-dT primer for $50 \mathrm{~min}$ at $42^{\circ} \mathrm{C}$. The cDNA was PCR amplified using Taq DNA Polymerase (Takara, Otsu, Japan) with primers specific for cyclin Dla, 5'-CAAATGGAGCTGCTCCTGG TG-3' (forward) and 5'-CTTCGATCTGCTCCTGGCAGG-3' (reverse); cyclin Dlb, 5'-CAAATGGAGCTGCTCCTGG TG-3' (forward) and 5'-TGGCACCAGCCTCGGCATTTC-3' (reverse) (13). PCR was performed for 35 cycles (cyclin D1b) or 30 cycles (cyclin Dla) consisting of denaturation at $94^{\circ} \mathrm{C}$ for $30 \mathrm{sec}$, annealing at $59^{\circ} \mathrm{C}$ for $30 \mathrm{sec}$ and extension at $72^{\circ} \mathrm{C}$ for $1 \mathrm{~min}$, followed by a final extension for $7 \mathrm{~min}$. CD44 primers were CD44-F (5'-AGAAGGTGTGGGCAGAAGAA-3' and CD44-R (5'-AAATGCACCATTTCCTGAGA-3'). Glyceraldehyde-3-phosphate dehydrogenase $(G A P D H)$ primers were GAPDH-5F (5'-ACCACAGTCCATGCCATCAC-3') and GAPDH-3R (5'-TCCACCACCCTGTTGCTGTA-3'). Moreover, 30 cycles of PCR was performed for GAPDH and $C D 44$ with annealing at $55^{\circ} \mathrm{C}$. The PCR products were separated by electrophoresis in a $2 \%$ agarose gel containing ethidium bromide and then photographed.

Transfection of siRNA oligonucleotides. The siRNA oligonucleotides for cyclin DIb and CD44 were synthesized by GeneDesign, Inc. (Osaka, Japan). siRNA sequences were 5'-CAAATGGAGCTGCTCCTGGTG-3' (sense) and 5'-TG GCACCAGCCTCGGCATTTC-3' (antisense) for $c y c l i n$ Dlb siRNA and 5'-AAAUGGUCGCUACAGCAUCTT-3' (sense) and 5'-GAUGCUGUAGCGACCAUUUTT-3' (antisense) for CD44 siRNA. A non-specific siRNA duplex (GeneDesighn) served as the control. The cells were transfected with siRNA for cyclin Dlb or CD44 using Lipofectamine ${ }^{\mathrm{TM}}$ RNAiMAX reagents (Invitrogen) according to the manufacturer's protocol.

Immunoblotting. Cells and tissues were lysed in Laemmli sodium dodecyl sulfate (SDS) buffer containing $62.5 \mathrm{mM}$ Tris- $\mathrm{HCl}$ (pH 6.8), 10\% glycerol, 5\% 2-mercaptoethanol, 2\% SDS, $0.01 \%$ bromophenol blue and $5 \mathrm{mM}$ ethylenediaminetetraacetic acid. A sample of the cell lysate was subjected to SDS-PAGE, and the separated proteins were electrotransferred to membrane filters (Immobilon-P; Millipore Corp., Billerica, MA, USA). After blocking with TBS-T [10 mM Tris- $\mathrm{HCl}$ ( $\mathrm{pH} 7.6), 150 \mathrm{mM}$ sodium chloride and $0.1 \%$ Tween-20] containing $5 \%$ bovine serum albumin (BSA), the filters were incubated overnight with the first antibody in TBS-T containing $2 \%$ BSA. The filters were washed with TBS-T and incubated for $1 \mathrm{~h}$ in horseradish peroxidase-conjugated anti-mouse or anti-rabbit IgG (GE Healthcare Bio-Science Corp., Arlington Heights, IL, USA) diluted to 1:20,000 in TBS-T containing 2\% BSA. After several washes with TBS-T, immunoreactivity was detected using the ECL system (GE Healthcare Bio-Science), according to the manufacturer's instructions. 
Antibodies. Anti- $\alpha$-tubulin (DM1A) monoclonal antibodies were purchased from Sigma-Aldrich (St. Louis, MO, USA). Anti-cyclin D1 rabbit monoclonal (92G2), anti-CD44 mouse monoclonal (156-3C11), anti-GAPDH rabbit monoclonal (D16H11), anti-N-cadherin rabbit monoclonal (D4R1H) and anti-caspase-3 rabbit monoclonal (D3R6Y) antibodies were purchased from Cell Signaling Technology (Tokyo, Japan). Anti-E-cadherin (36/E-cadherin) and anti-Rb (G3-245) mouse monoclonal antibodies were purchased from BD Biosciences, whereas anti-vimentin (C9) mouse monoclonal antibody was purchased from Santa Cruz Biotechnology Inc. (Santa Cruz, CA, USA).

Tumorigenicity assay in nude mice. The use of animals in the experimental protocols was reviewed and approved by the Management Committee of the Research Center for Animal Life Science at Shiga University of Medical Science. The animals were housed in a specific pathogen-free room with controlled temperature $\left(20-22^{\circ} \mathrm{C}\right)$ and humidity $(50-60 \%)$ and a preset light-dark cycle $(12: 12 \mathrm{~h})$. They were allowed ad libitum access to food (CE-2; CLEA Japan, Inc., Tokyo, Japan) and water. The right dorsal flank of each 6-week-old BALB/c-nu/nu female nude mouse was injected subcutaneously with $5 \times 10^{6}$ SBT31A cells. After establishing palpable tumors $\left(\sim 50 \mathrm{~mm}^{3}\right)$, external tumor volume was determined on days $0,4,8,12$ and 16 . A total of 12 mice carrying palpable tumors were randomized into two groups of 6 mice. siRNA complexes were prepared using the Invivofectamine ${ }^{\circledR} 3.0$ reagent (Invitrogen) and siRNA duplex solution (cyclin DIb and control siRNA) according to the manufacturer's protocol. Each siRNA complex was injected into the tumor of each mouse at a dose of $5 \mu \mathrm{g}$ on days $0,4,8$ and 12. An injection volume of $100 \mu \mathrm{l}$ was prepared for each tumor. Tumor volume was calculated using the formula $\mathrm{A} \times \mathrm{B} \times \mathrm{C} / 2$, where $\mathrm{A}, \mathrm{B}$ and $\mathrm{C}$ represent the diameters of length, height and width, respectively. Resected tumors that developed on the back of the mice were fixed with $10 \%$ formalin in PBS for $4 \mathrm{~h}$ and embedded in paraffin. Serial $3 \mu \mathrm{m}$ sections were used for histological evaluation using hematoxylin and eosin staining. For the detection of apoptotic cells in tumor tissues, terminal deoxynucleotidyl transferase dUTP nick end labeling (TUNEL) assays were performed using the Apoptosis in situ Detection kit (Wako Pure Chemical Industries, Osaka, Japan) according to the manufacturer's protocol (17).

DAPI (4',6-diamidino-2-phenylindole) staining. The SBT31A cells were stained with DAPI $(10 \mathrm{mg} / \mathrm{ml}$ in PBS) for $30 \mathrm{~min}$, followed by fixation with $4 \%$ paraformaldehyde for $15 \mathrm{~min}$ in the dark. Cell apoptotic morphology was observed using a fluorescence microscope (BX61; Olympus, Inc., Tokyo, Japan).

Three-dimensional (3D) spheroid formation assay. 3D spheroid formation was examined by culturing the cells in 96-well V plates (PrimeSurface ${ }^{\circledR}$; Sumitomo Bakelite Co., Ltd., Tokyo, Japan). In brief, $1 \times 10^{3}$ cells were inoculated in triplicate into each well containing RPMI-1640 medium supplemented with $10 \% \mathrm{FCS}$ and incubated at $37^{\circ} \mathrm{C}$ in a humidified $5 \% \mathrm{CO}_{2}$ atmosphere. After 3 days of incubation, spheroid formation was photographed using phase contrast microscopy (x4 magnification; Olympus). The CellTiter-Glo ${ }^{\circledR}$
3D Cell Viability Assay (Promega, Madison, WI, USA) was then used to determine the number of viable cells in the 3D cell culture based on quantitation of the ATP present according to the manufacturer's protocol. The Caspase-Glo ${ }^{\circledR} 3 / 7$ assay (Promega) was used to measure caspase- 3 and -7 activities according to the manufacturer's protocol. The morphology of the spheroids was observed using a microscope (Diaphot 30D; Nikon, Inc., Tokyo, Japan).

$3 D$ spheroid invasion assay. For spheroid generation, cell suspensions of $100 \mu \mathrm{l} /$ well at optimized densities $\left(1 \times 10^{3}\right.$ cells $/ \mathrm{ml}$ ) were dispensed into 96-well U-plates (PrimeSurface ${ }^{\circledR}$ Sumitomo Bakelite). Plates were incubated for 3 days at $37^{\circ} \mathrm{C}$ in a humidified $5 \% \mathrm{CO}_{2}$ atmosphere. Where indicated, optimal $3 \mathrm{D}$ structures were achieved by adding $100 \mu \mathrm{l}$ of Matrigel (Corning Life Sciences B.V, Amsterdam, The Netherlands). After incubation, spheroid images were captured using an Olympus IX 70 inverted microscope (Olympus) equipped with a CCD camera and imported into the Image-Pro Plus 6.2 Analyzer software (Media Cybernetics, Inc., Rockville, MD, USA). Thereafter, maximal radial distances for invaded cells were calculated using Image $1.51 \mathrm{~h}$.

Statistical analyses. All quantitative data are presented as means \pm standard deviations (SD). Welch's t-test was used for statistical analyses. The statistical analyses were performed using the $\mathrm{R}$ statistical software package, version 2.6.2 (R Foundation for Statistical Computing, Vienna, Austria). Significance was set at $\mathrm{P}<0.05$.

\section{Results}

Effect of cyclin Dlb knockdown on cell proliferation and invasiveness. To examine the role of cyclin Dlb on the expression of malignant phenotypes in bladder carcinogenesis, we suppressed the expression of cyclin D1b in human bladder cancer cell lines, T24 and SBT31A, by introducing cyclin Dlb siRNA. Cyclin Dlb siRNA markedly suppressed the expression of cyclin Dlb transcripts compared to the no-treatment control and control siRNA in both cell lines $24 \mathrm{~h}$ after transfection (Fig. 1A). Protein levels of cyclin D1(a) were not affected by cyclin Dlb siRNA treatment (Fig. 1B), indicating that such treatment was able to specifically suppress cyclin DIb expression under these experimental conditions. To evaluate the anti-oncogenic effects of cyclin Dlb siRNA in these cell lines, we initially compared cell proliferation and in vitro cell invasiveness after treatment with control and cyclin Dlb siRNAs. As shown in Fig. 1C and D, cyclin Dlb siRNA significantly suppressed the proliferation of SBT31A and T24 cells compared to control siRNA. Cell invasiveness of both cell lines was also significantly suppressed by cyclin Dlb siRNA (Fig. 1E-H).

Cell cycle arrest in the G1 phase and induction of apoptosis by cyclin DIb siRNA treatment. To further assess the effects of cyclin Dlb siRNA on these cancer cells, we performed FACS analyses in cells treated with control and cyclin DIb siRNAs $48 \mathrm{~h}$ after transfection. As shown in Fig. 2A, cyclin Dlb siRNA treatment significantly decreased the population of the $S$ phase and increased that of the G1 phase in T24 cells compared 
A

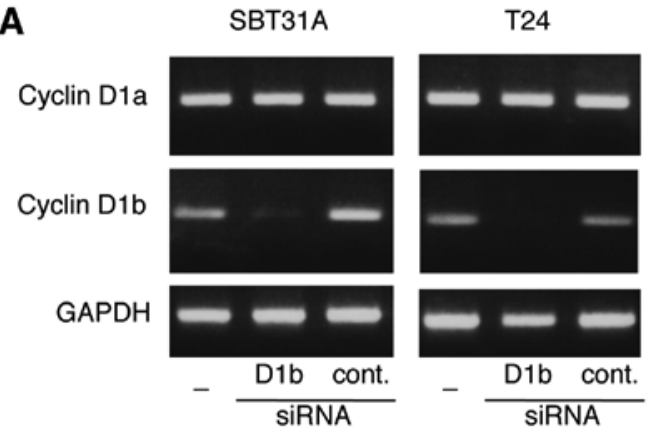

B

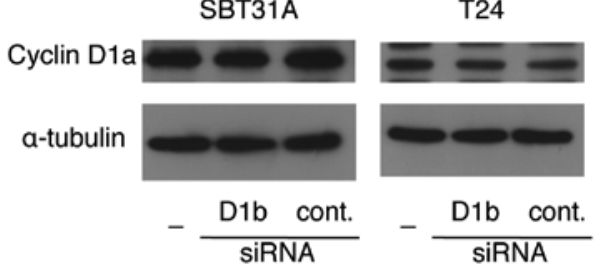

C

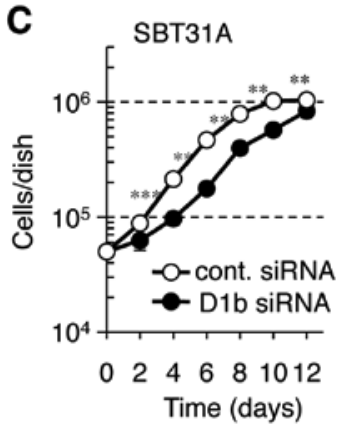

G

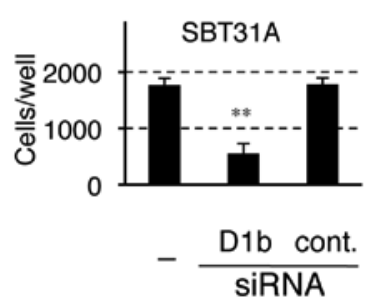

D

D T24

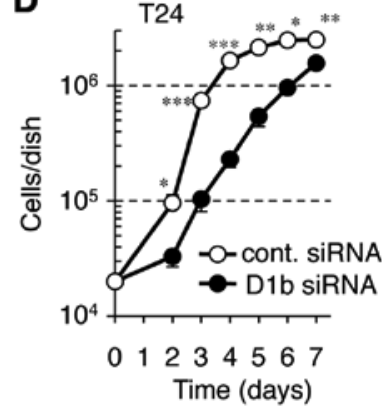

H

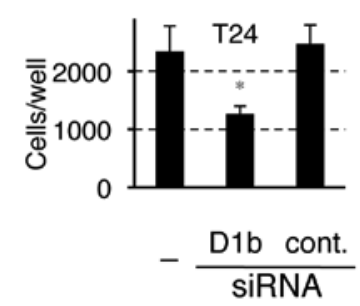

E

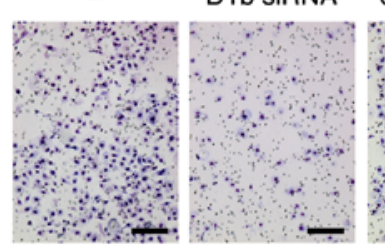

cont. SiRNA

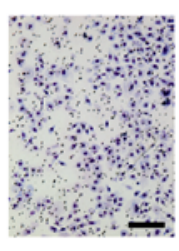

F

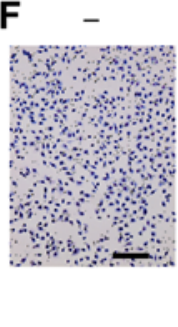

D1b siRNA

cont. siRNA
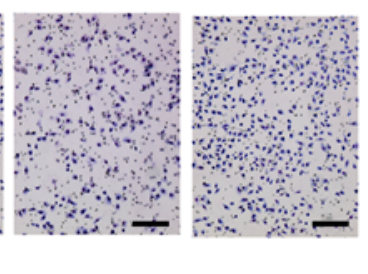

Figure 1. Anti-oncogenic effects of cyclin D1b siRNA in SBT31A and T24 cells. (A) Expression of $c y c l i n$ D1b mRNA in SBT31A and T24 cells transfected with control and cyclin D1b siRNAs. GAPDH was used as an internal control. (B) Expression of cyclin D1a protein in SBT31A and T24 cells transfected with control and cyclin D1b siRNAs. $\alpha$-tubulin was used as an internal control. (C) Growth curves of SBT31A cells transfected with control and $c y c l i n$ D1b siRNAs. The open and closed circles indicate control and cyclin DIb siRNAs, respectively. The values represent the mean \pm SD. (D) Growth curves of T24 cells transfected with control and cyclin D1b siRNAs. The open and closed circles indicate control and $c y c l i n$ D1b siRNAs, respectively. The values represent the mean \pm SD. (E) Images of the SBT31A cells invading through Matrigel. (F) Images of the T24 cells invading through Matrigel. Bars indicate $200 \mu \mathrm{m}$. Photographs were taken at a magnification of x100. (G) Effects of cyclin D1b siRNA on cell invasiveness of SBT31A cells. Each sample was assayed in triplicate, and the bar represents the mean \pm SD. (H) Effects of cyclin D1b siRNA on cell invasiveness of T24 cells. Each sample was assayed in triplicate, and the bar represents the mean $\pm \mathrm{SD} .{ }^{*} \mathrm{P}<0.05,{ }^{* *} \mathrm{P}<0.01$ and ${ }^{* * *} \mathrm{P}<0.001$ compared with no treatment, respectively. SD; standard deviation.

to the no-treatment control and control siRNA. Given that the $\mathrm{Rb}$ protein is a key regulator in the G1-S transition of the cell cycle, we examined the change in $\mathrm{Rb}$ phosphorylation through immunoblot analysis with specific antibodies of Rb. As shown in Fig. 2B, cyclin Dlb siRNA decreased upper hyper-phosphorylated $\mathrm{Rb}$ and increased lower hypophosphorylated Rb compared to control siRNA in T24 cells, indicating that regulation of $\mathrm{Rb}$ phosphorylation plays a critical role in cell cycle suppression, at least in T24 cells. In contrast to $\mathrm{T} 24$ cells, $\mathrm{Rb}$ expression was not detected in SBT31A cells (Fig. 2B), suggesting that other mechanisms are involved in the suppression of cell proliferation by $c y c l i n$ DIb siRNA. FACS analyses showed that the sub-G1 population was significantly increased in SBT31A cells treated with cyclin Dlb siRNA $(14.3 \pm 2.2 \%)$ compared to the no-treatment control and control siRNA cells $(7.5 \pm 1.0 \%)$, suggesting the induction of apoptosis by knockdown of cyclin Dlb (Fig. 2A). The increase in the sub-G1 fraction was hardly detected in T2 4 cells transfected with both control and cyclin D1b siRNAs. To confirm the induction of apoptosis by cyclin Dlb siRNA in
SBT31A cells, we performed nuclear staining with DAPI and immunoblot analysis with anti-caspase-3 antibody. As shown in Fig. 2C and D, the percentage of morphologically aberrant nuclei with nuclear fragmentation was significantly increased in SBT31A cells treated with cyclin D1b siRNA $(24.3 \pm 2.1 \%)$ compared to those treated with control siRNA $(12.5 \pm 1.7 \%)$ or those that had no treatment $(8.1 \pm 1.1 \%)$. Activation of caspase-3 (cleaved lower band) was also detected in SBT31A cells, but not T24 cells, treated with cyclin DIb siRNA (Fig. 2E). These results indicated that, in SBT31A cells, the induction of apoptosis plays a critical role in the suppression of cell proliferation by knockdown of cyclin Dlb.

Effects of cyclin Dlb knockdown on 3D spheroid formation. 3D spheroid formation models are recently being used in cancer research to more closely mimic the environment associated with tumors (18). We also examined the anti-oncogenic effects of cyclin Dlb siRNA in this multicellular tumor spheroid model. After cyclin Dlb siRNA transfection for $24 \mathrm{~h}, 1 \times 10^{3}$ SBT31A and T24 cells were inoculated into 96-well plates for 
A
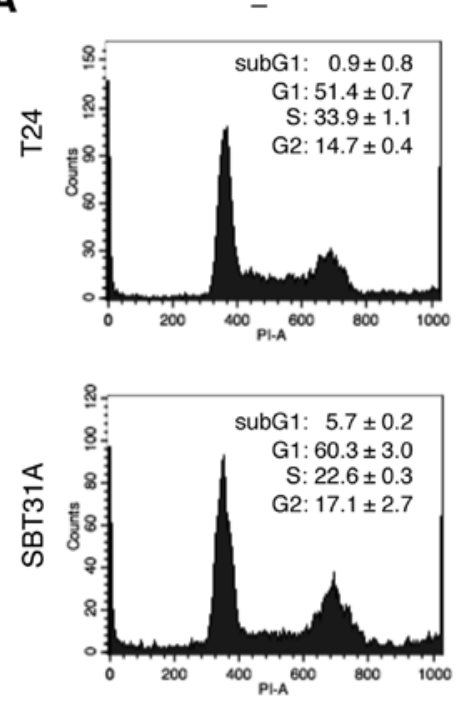

B

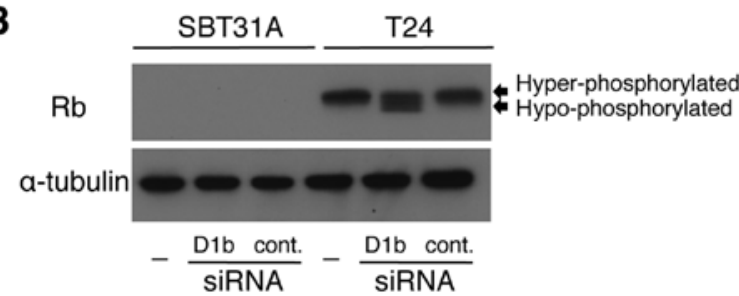

D1b siRNA
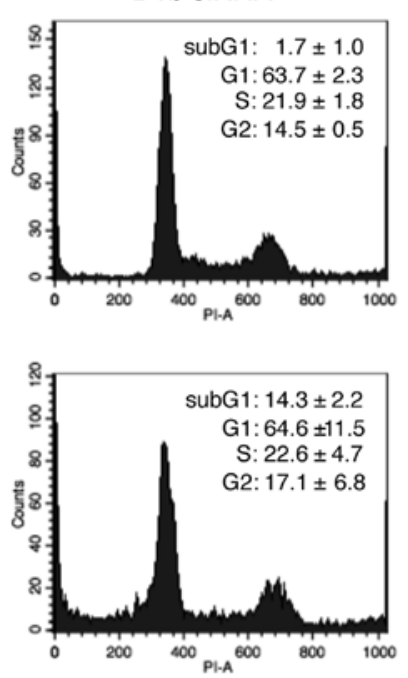

cont. siRNA
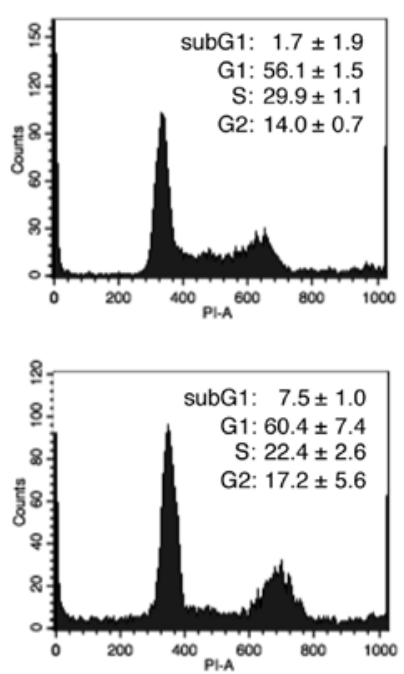

C

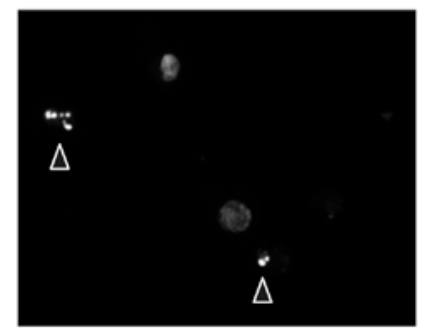

D

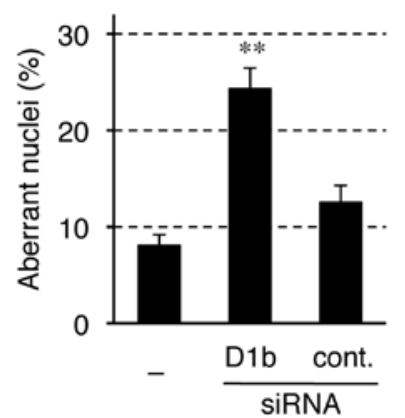

E

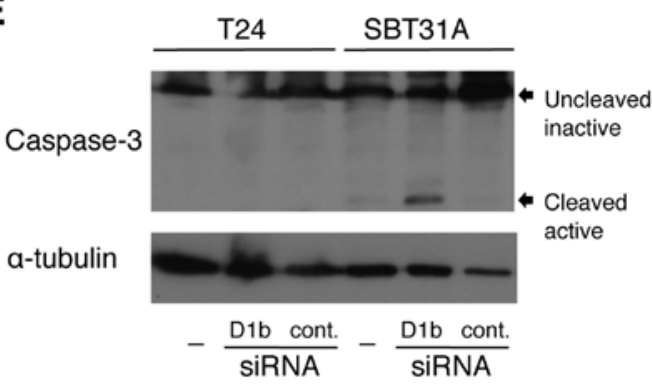

Figure 2. Cell cycle and apoptosis analyses of SBT31A and T24 cells transfected with control and cyclin D1b siRNAs. (A) FACS analyses of SBT31A and T24 cells transfected with control and cyclin D1b siRNAs. (B) Rb phosphorylation in SBT31A and T24 cells transfected with $c y c l i n$ D1b siRNA. $\alpha$-tubulin was used as an internal control. (C and D) Induction of apoptosis in SBT31A cells by cyclin D1b siRNA. (C) Morphological examination of SBT31A cells transfected with cyclin Dlb siRNA. At $72 \mathrm{~h}$ after transfection with $c y c l i n$ Dlb siRNA, the cells were stained with DAPI (10 mg/ml). Fluorescence was observed under a fluorescence microscope. The arrowheads indicate aberrant nuclei. (D) Percentage of aberrant nuclei with nuclear fragmentation. Each bar indicates the mean $\pm \mathrm{SD} .{ }^{* *} \mathrm{P}<0.01$ compared with no treatment. (E) Activation of caspase-3 in SBT31A and T24 cells transfected with $c y c l i n$ D1b siRNA. $\alpha$-tubulin was used as an internal control.

3D spheroid formation. The spheroid-forming ability of these cells was examined after incubation for 3 days in complete culture medium. As shown in Fig. 3A, spheroids grew dim in SBT31A cells treated with cyclin DIb siRNA, while outer layer spheroid formation was inhibited in T24 cells treated similarly. These inhibitory changes in spheroid formation were not observed in control siRNA treatment. The number of viable cells in these spheroids was assessed using ATP activity in both cell lines. As shown in Fig. 3B and C, this was significantly decreased by cyclin Dlb siRNA compared to controls (left panels). In addition, caspase-3/-7 activities (middle panels) and the caspase-3/-7/ATP activity ratio (right panels) were increased by cyclin DIb siRNA in SBT31A cells (Fig. 3B) but not in T24 cells (Fig. 3C). These results revealed that apoptosis also contributed to the suppression of 3D spheroid formation by cyclin DIb siRNA in SBT31A cells. On the other hand, although the number of viable cells significantly decreased in T24 cells treated with $c y c l i n$ DIb siRNA, caspase-3/-7 activity similarly decreased in these cells (Fig. 3C). This was probably because the decrease in caspase-3/-7 activities reflects the decrease in viable cell number of T24 spheroids treated with cyclin DIb siRNA, revealing that knockdown of cyclin DIb suppressed cell proliferation of T24 cells in 3D spheroid culture. These 3D spheroid culture results are consistent with those of adhesive cultures in Figs. 1 and 2.

Effects of cyclin D1b knockdown on cancer stemness and epithelial-mesenchymal transition (EMT)-related 
A

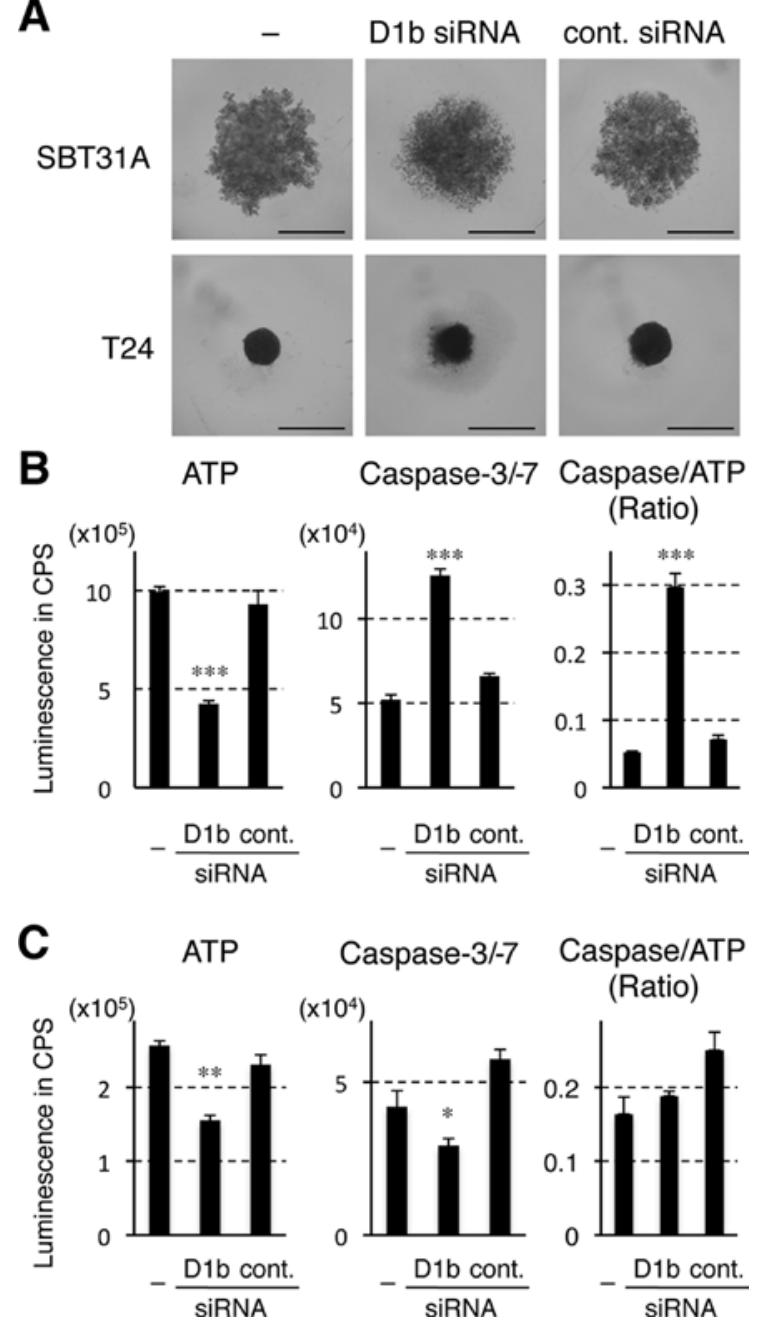

Figure 3. Effect of cyclin Dlb siRNA on 3D spheroid formation of SBT31A and T24 cells. (A) Changes in the morphology of spheroids by transfection of cyclin DIb siRNA. Scale bars indicate $50 \mu \mathrm{m}$. (B) Effects of cyclin Dlb siRNA on ATP and caspase-3/-7 activities of formed spheroids in SBT31A cells. ATP activity (left), caspase-3/-7 activity (middle) and the caspase-3/-7/ATP activity ratio (right) are shown. The bars indicate the mean \pm SD. (C) Effects of cyclin DIb siRNA on ATP activity and caspase-3/-7 activity of formed spheroids in T24 cells. ATP activity (left), caspase-3/-7 activity (middle) and the caspase (3/7)/ATP activity ratio (right) are shown. The bars indicate the mean \pm SD. ${ }^{*} \mathrm{P}<0.05,{ }^{* *} \mathrm{P}<0.01$ and ${ }^{* * * *} \mathrm{P}<0.001$ compared with no treatment, respectively.

invasiveness of T24 cells. As given that 3D spheroid formation is considered to be one of the cancer stem cell phenotypes (19), we investigated alterations in the expression of CD44, a stem cell marker of human bladder cancer, in SBT31A and T24 cells treated with cyclin Dlb siRNA (Fig. 4A). Although the expression of CD44 was hardly detected in SBT31A cells, it was evidently detected and suppressed by cyclin DIb siRNA in T24 cells, suggesting that cyclin Dlb knockdown suppresses the cancer stem cell phenotype via CD44 in T24 cells. To assess the role of CD44 in the suppression of 3D spheroid formation by cyclin Dlb siRNA, CD44 expression was knocked down by $C D 44$ siRNA in T24 cells (Fig. 4B). As shown in Fig. 4C, CD44 knockdown hardly affected ATP activity in T24 spheroid culture. However, CD44 siRNA changed the morphology of T24 spheroids (Fig. 4D). Given that the phenotype of cancer stem cells is closely correlated with EMT (20), we examined the expression of EMT-related proteins in T24 cells treated with cyclin Dlb siRNA. As shown in Fig. 4E and F, the expression of N-cadherin, an EMT protein, was significantly decreased $(\sim 50 \%)$ by cyclin $\mathrm{Dlb}$ knockdown in T24 cells. On the other hand, the expression of E-cadherin was faintly detected and not altered by cyclin DIb siRNA (Fig. 4E). The expression of vimentin was not affected by $C D 44$ siRNA (Fig. 4E). Similar results were obtained in T24 cells treated with $C D 44$ siRNA (Fig. 4B). To exclude the possibility that $C D 44$ siRNA suppress the expression of N-cadherin via suppression of cyclin Dlb mRNA, we examined the effect of $C D 44$ siRNA on the expression of cyclin D1b by RT-PCR. As shown in Fig. 4G, CD44 siRNA did not suppress the expression of cyclin DIb mRNA, indicating that cyclin D1b acts upsteam of CD44 for suppression of $\mathrm{N}$-cadherin expression. These results indicate that knockdown of cyclin Dlb suppresses N-cadherin expression via CD44. To further evaluate these correlations, we examined the invasion activity (invadopodia) of spheroid cells treated with CD44 and cyclin Dlb siRNAs using T24 spheroids embedded in Matrigel. As shown in Fig. $4 \mathrm{H}$ and I, the invadopodia formation of T24 spheroid cells was suppressed by $C D 44$ and cyclin Dlb siRNAs compared to control siRNA. Taken together, these results indicate that knockdown of cyclin Dlb is able to suppress malignant phenotypes of T24 cells, including cancer stemness and invasiveness, via the regulation of CD44 and $\mathrm{N}$-cadherin expression.

Suppression of tumor growth in vivo by cyclin DIb siRNA. To assess whether cyclin Dlb siRNA is able to suppress in vivo tumor growth of human bladder cancer cells expressing cyclin Dlb, the tumorigenicity assay was performed in nude mice. Given that SBT31A cells can efficiently form tumors through subcutaneous injection in nude mice, we used these cells in our in vivo tumor model (16). T24 cells did not form tumors in nude mice under these experimental conditions. Upon reaching a palpable volume $\left(\sim 50 \mathrm{~mm}^{3}\right)$, control and cyclin Dlb siRNAs were injected into the tumors using the Invivofectamine reagent. Nude mice with palpable tumors were randomly separated into two groups of six. There was no significant difference in tumor volume between cyclin Dlb $\left(55.8 \pm 11.3 \mathrm{~mm}^{3}\right)$ and control $\left(58.3 \pm 14.1 \mathrm{~mm}^{3}\right)$ siRNA groups $(\mathrm{P}=0.7415)$. As shown in Fig. 5A and B, tumor growth was significantly inhibited in SBT31A cells treated with cyclin DIb siRNA compared to those treated with control siRNA. The resected tumors of the cyclin Dlb siRNA-treated group (178 $\pm 64 \mathrm{mg})$ were significantly smaller than those of the control siRNA-treated group $(408 \pm 86$ mg, $\mathrm{P}=0.0003$ ) (Fig. 5C). Histopathological (Fig. 5D-G) and TUNEL (Fig. 5H and I) examinations showed that apoptotic cells were significantly increased in tumor tissues treated with cyclin DIb siRNA. These results indicate that cyclin DIb siRNA is able to inhibit the in vivo growth of SBT31A tumors by inducing apoptosis. Hence, cyclin Dlb siRNA will serve as a novel therapeutic agent for the treatment of bladder cancers that express cyclin Dlb.

\section{Discussion}

In the present study, we investigated the anti-oncogenic effects of $c y c l i n$ Dlb siRNA on human bladder cancer cell 
A

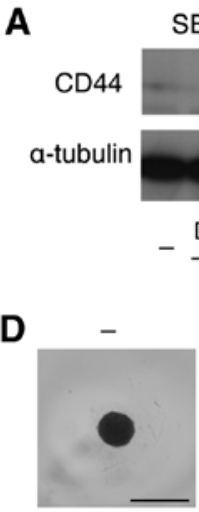

E

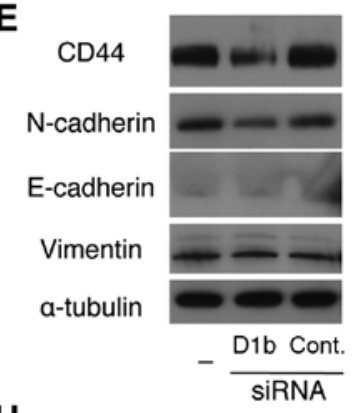

H

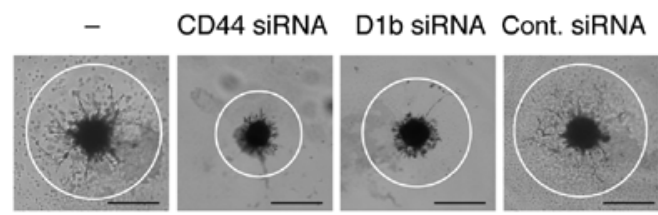

B

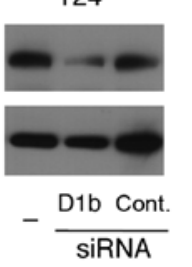

\section{CD44}

$\mathrm{N}$-cadherin

E-cadherin

Vimentin

a-tubulin

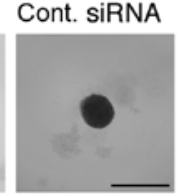

F N-cadherin/a-tubulin

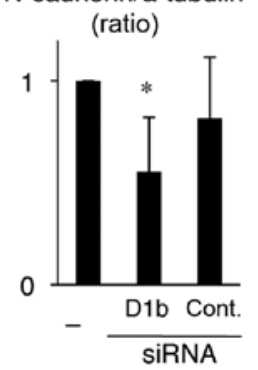

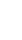

C

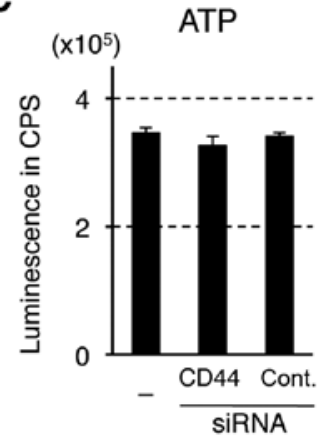

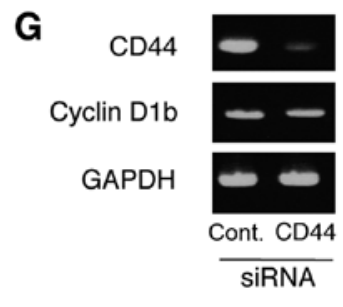

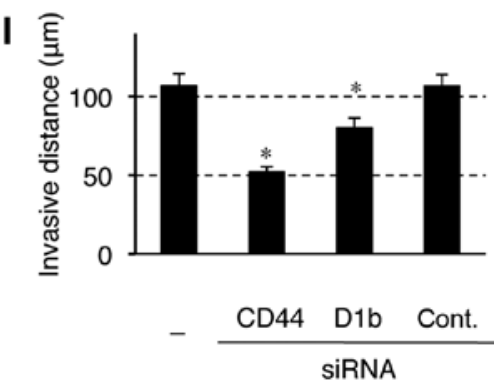

Figure 4. Effect of cyclin D1b siRNA on the expression of CD44 and N-cadherin in T24 cells. (A) Effects of cyclin D1b siRNA on the expression of CD44 in SBT31A and T24 cells. $\alpha$-tubulin was used as an internal control. (B) Effects of $C D 44$ siRNA on the expression of EMT-related proteins in T24 cells. $\alpha$-tubulin was used as an internal control. (C) Effects of $C D 44$ siRNA on cell viability of spheroids formed by T24 cells. The bars indicate the mean \pm SD. (D) Morphological changes of 3D spheroids by transfection of CD44 siRNA in T24 cells. Scale bars indicate $50 \mu \mathrm{m}$. (E) Effects of $c y c l i n$ D1b siRNA on the expression of EMT-related proteins in T24 cells. $\alpha$-tubulin was used as an internal control. (F) The N-cadherin/ $\alpha$-tubulin ratio. The values were calculated by densitometry. Each sample was assayed in triplicate, and the bar represents the mean $\pm \mathrm{SD} .{ }^{*} \mathrm{P}<0.05$ compared with no treatment. (G) Expression of $c y c l i n$ D1b and CD44 mRNA in T24 cells transfected with control and CD44 siRNAs. GAPDH was used as an internal control. (H) The invadopodia formation in 3D spheroids of T24 cells transfected with CD44 and cyclin D1b siRNAs. White circles indicate the maximum radial distance traveled by invaded cells. Scale bars indicate $50 \mu \mathrm{m}$. (I) Quantification of the invasive distance traveled by T24 cells transfected with CD44 and $c y c l i n$ D1b siRNAs. Each sample was assayed in triplicate, and the bar represents the mean $\pm \mathrm{SD}$. ${ }^{*} \mathrm{P}<0.05$ compared with no treatment.

lines, which express cyclin Dlb, and found that it had the ability to effectively suppress in vitro and in vivo malignant phenotypes of bladder cancer cells. Knockdown of $c y c l i n$ DIb by specific siRNAs significantly suppressed proliferation and in vitro invasiveness of both SBT31A and T24 cells. Cell cycle analyses revealed that cyclin DIb siRNA inhibited the G1-S transition in T24 cells. On the other hand, cyclin DIb siRNA induced an increase in the sub-G1 fraction after FACS analysis, an increase in morphological aberrant nuclei with nuclear fragmentation, and the activation of caspase-3 in SBT31 A cells, indicating induction of apoptosis. Although cyclin D1b siRNA did not induce apoptosis in T24 cells, it suppressed the expression of a cancer stem cell marker, CD44, in T24 cells. Moreover, 3D spheroid formation was also inhibited by cyclin DIb siRNA in SBT31A and T24 cells. Apoptosis was induced in spheroids of SBT31A cells but not in those of T24 cells. Knockdown of CD44 or cyclin D1b suppressed invadopodia formation of spheroids and the expression of $\mathrm{N}$-cadherin in T24 cells. Taken together, these results demonstrate that knockdown of cyclin Dlb successfully suppresses malignant phenotypes of human bladder cancer cells via multiple mechanisms, such as suppression of the cell cycle, induction of apoptosis and suppression of EMT and cancer stemness.

Although the expression of Rb and CD44 proteins was not detected in SBT31A cells, they were evidently expressed in T24 cells, suggesting that the difference in the expression of these proteins is due to the different responses of these cell lines to introduction of cyclin Dlb siRNA. In the HT1197 bladder cancer cell line, wherein the expression of $c y c l i n$ Dlb mRNA was not detected, knockdown of cyclin Dlb neither induced apoptosis nor suppressed cell proliferation (data not shown), indicating that the responses of SBT31A and T24 cells to $c y c l i n$ DIb siRNA depend on the expression of cyclin DIb. 
A

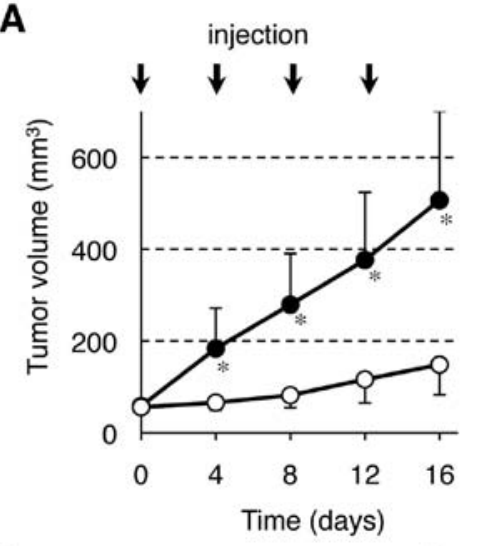

D

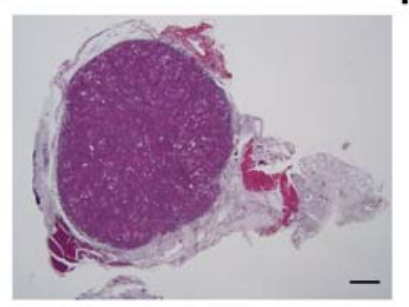

E

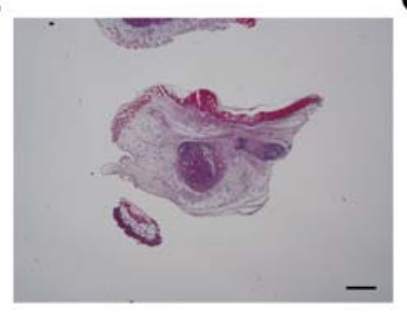

B

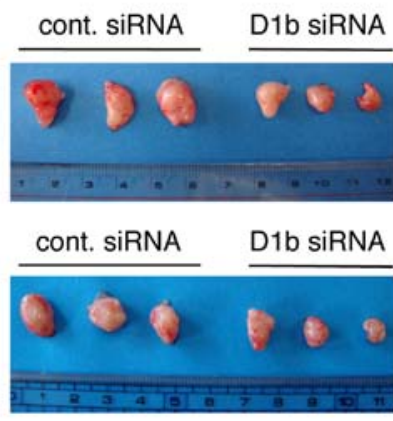

$\mathbf{F}$

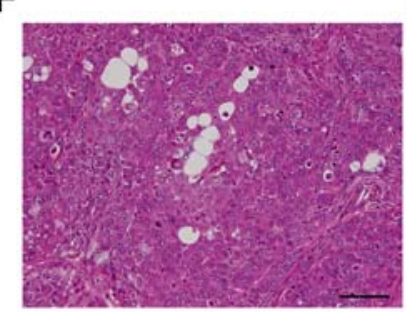

G

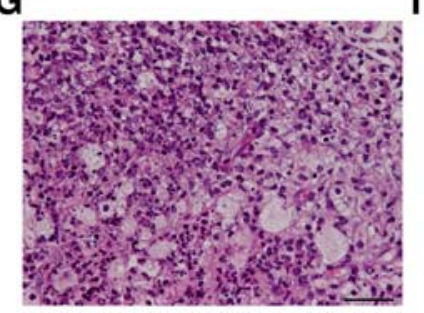

H

I
C

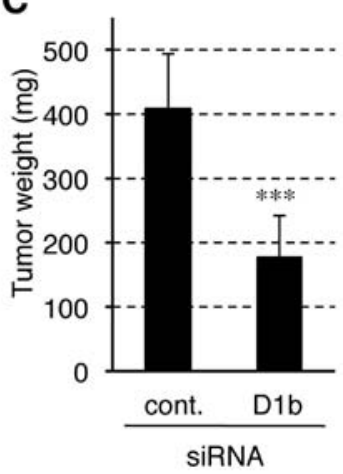

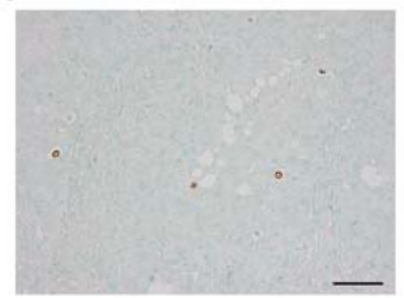

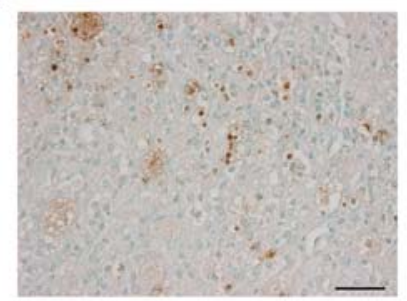

Figure 5. Effects of cyclin D1b siRNA on tumor growth of SBT31A cells in nude mice. (A) Effects of cyclin D1b siRNA on tumor growth of SBT31A cells. The arrows indicate the days in which control and cyclin Dlb siRNAs were injected into the tumors of nude mice. The closed and open circles represent control and cyclin Dlb siRNAs, respectively. The values indicate the mean \pm SD. ${ }^{*} \mathrm{P}<0.05$ compared with no treatment. (B) Representative tumor samples from each group. (C) The weight of resected tumors. The bars indicate the mean $\pm \mathrm{SD}$. ${ }^{* * *} \mathrm{P}<0.001$ compared with no treatment. (D-G) H\&E staining of tumor tissues injected with control (D and F) and cyclin Dlb (E and G) siRNAs. Scale bars indicate $1 \mathrm{~mm}$ (D and E, magnification, $\mathrm{x} 40$ ) and $100 \mu \mathrm{m}$ (F and G, magnification, x200). (H and I) TUNEL staining of tumor tissues injected with control (H) and cyclin Dlb (I) siRNAs. Scale bars indicate $100 \mu$ m, magnification, x200.

$\mathrm{Rb}$ has been shown to be deleted in $37 \%$ of invasive human bladder cancers (21-23). Although $\mathrm{Rb}$ is known to regulate the cell cycle and control apoptosis, it has also been shown to be involved in the process of malignant progression, including EMT and metastasis of many human cancers (24-26). In fact, SBT31A cells, lacking Rb, had a higher ability to induce anchorage-independent growth and form tumors in nude mice compared to $\mathrm{T} 24$ cells. $\mathrm{Rb}$ serves as a critical point in the regulation of the G1-S transition of the cell cycle $(24,25)$. In the presence of $\mathrm{Rb}$, cyclin D1b accelerates the cell cycle progression via the Akt-Erk pathway (15). In this case, knockdown of cyclin Dlb causes the suppression of cell proliferation. On the other hand, in the absence of Rb, cyclin D1b may act to suppress apoptosis caused by the dysregulation of the cell cycle. Thus, knockdown of cyclin Dlb could induce apoptosis. Nevertheless, in this study, knockdown of cyclin D1b suppressed cancer phenotypes irrespective of $\mathrm{Rb}$ expression, indicating that knockdown of cyclin Dlb is able to confer its therapeutic ability in most invasive human bladder cancers.

Tumor growth of SBT31A cells in nude mice was significantly inhibited by $c y c l i n$ DIb siRNA via the induction of apoptosis. In breast cancer, knockdown of cyclin Dlb was shown to suppress in vivo tumor formation by inducing apoptosis (27). However, in the present study, we further found the ability of cyclin Dlb siRNA to suppress the stemness and invasiveness of bladder cancer cells via suppression of CD44 and N-cadherin expression in T24 cells. CD44, a multi-structural and multifunctional transmembrane glycoprotein, was identified as a receptor for hyaluronan and was found to be closely associated with the development of a variety of solid tumors (20,28-32). Furthermore, the elevated expression of CD44 has been shown to be correlated with the phenotypes of cancer stem cells and EMT in various human cancers, such as those of the breast, prostate, pancreas and bladder (20). Although CD44 variants, such as v6 and v9, have been reported to play a role in these cancers (20), expression of these variants was not detected by RT-PCR in T24 and SBT31A cells (data not shown). We also demonstrated that knockdown of CD44 suppressed cell invasiveness of spheroids and expression of N-cadherin in T24 cells, suggesting that cyclin D1b regulates the expression of CD44 thereby affecting invasion and cancer stemness. As for the induction of EMT, downregulation of E-cadherin and upregulation of $\mathrm{N}$-cadherin have been shown to play critical roles in a variety 
of human cancers (33-38). Notably, elevated expression of $\mathrm{N}$-cadherin has been reported to play a critical role in human bladder carcinogenesis $(39,40)$. In T24 cells, the expression of E-cadherin was hardly detected, and cyclin Dlb siRNA was not able to increase it. On the other hand, the expression of N-cadherin was significantly suppressed by cyclin Dlb and $C D 44$ siRNAs, suggesting its vital role in suppressing the invasion of bladder cancers. These results indicate that the expression of cyclin D1b plays a critical role in the maintenance of cancer stemness and EMT via regulation of CD44 and $\mathrm{N}$-cadherin expression.

Knockdown of cyclin $D 1 b$ promoted apoptosis of cyclin D1b-expressing SBT31A bladder cancer cells. We also revealed that cyclin $D 1 b$ siRNA inhibited tumor growth of cancer cells in nude mice by intratumoral injections, demonstrating the therapeutic potential of cyclin DIb siRNA for the treatment of $c y c l i n D l b$-expressing bladder cancers. To use this treatment in clinical studies, the siRNAs have to be modified to reduce its sensitivity to nucleases and increase its cellular uptake (41). In bladder cancer cases, transurethral endoscopic surgery is initially applied for non-muscle invasive bladder cancers. However, after endoscopic surgery, bladder cancers have an intravesical recurrence rate of $50-70 \%$ (42). The intravesical instillation of a chemotherapeutic agent is widely applied to decrease the risk of recurrence in patients with bladder cancers after the endoscopic surgery (43). In addition, it is possible to directly administer high concentrations of anticancer agents through transurethral therapy, in contrast to systemic therapy, because the anticancer agents can be administered to the intravesical space by transurethral catheterization. Indeed, Nogawa et al (44) demonstrated that intravesical administration of Polo-like kinase-1-targeted siRNA/cationic liposomes inhibited cancer growth in mouse orthotopic bladder cancer models. It is assumed that these characteristics of the transurethral approach will be beneficial to the practical application of the in vivo siRNA therapy in human bladder cancers.

\section{Acknowledgements}

We would like to thank Ms. Akiyo Ushio (Shiga University of Medical Science) for technical assistance. The present study was supported by the KAKENHI, Japan Society for the Promotion of Scientific Research, Grant nos. 19591843 , 21590437, 24590480 and 23590457).

\section{References}

1. Knudsen KE, Diehl JA, Haiman CA and Knudsen ES: Cyclin D1: Polymorphism, aberrant splicing and cancer risk. Oncogene 25: 1620-1628, 2006.

2. Sherr CJ: D-type cyclins. Trends Biochem Sci 20: 187-190, 1995.

3. Lukas J, Parry D, Aagaard L, Bartkova J, Strauss M, Peters G and Bartek J: Retinoblastoma-protein-dependent cell-cycle inhibition by tumour suppressor p16. Nature 375: 503-506, 1995.

4. Musgrove EA, Caldon CE, Barraclough J, Stone A and Sutherland RL: Cyclin D as a therapeutic target in cancer. Nat Rev Cancer 11: 558-572, 2011.

5. Hosokawa Y, Gadd M, Smith AP, Koerner FC, Schmidt EV and Arnold A: Cyclin D1 (PRAD1) alternative transcript b: Fulllength cDNA cloning and expression in breast cancers. Cancer Lett 113: 123-130, 1997.
6. Lu F, Gladden AB and Diehl JA: An alternatively spliced cyclin $\mathrm{D} 1$ isoform, cyclin D1b, is a nuclear oncogene. Cancer Res 63: 7056-7061, 2003.

7. Solomon DA, Wang Y, Fox SR, Lambeck TC, Giesting S, Lan Z, Senderowicz AM, Conti CJ and Knudsen ES: Cyclin D1 splice variants. Differential effects on localization, RB phosphorylation, and cellular transformation. J Biol Chem 278: 30339-30347, 2003.

8. Burd CJ, Petre CE, Morey LM, Wang Y, Revelo MP, Haiman CA, Lu S, Fenoglio-Preiser CM, Li J, Knudsen ES, et al: Cyclin Dlb variant influences prostate cancer growth through aberrant androgen receptor regulation. Proc Natl Acad Sci USA 103: 2190-2195, 2006.

9. Holley SL, Parkes G, Matthias C, Bockmühl U, Jahnke V, Leder K, Strange RC, Fryer AA and Hoban PR: Cyclin D1 polymorphism and expression in patients with squamous cell carcinoma of the head and neck. Am J Pathol 159: 1917-1924, 2001.

10. Howe D and Lynas C: The cyclin D1 alternative transcripts [a] and $[\mathrm{b}]$ are expressed in normal and malignant lymphocytes and their relative levels are influenced by the polymorphism at codon 241. Haematologica 86: 563-569, 2001.

11. Bala S and Peltomäki P: CYCLIN D1 as a genetic modifier in hereditary nonpolyposis colorectal cancer. Cancer Res 61: 6042-6045, 2001.

12. Paronetto MP, Cappellari M, Busà R, Pedrotti S, Vitali R, Comstock C, Hyslop T, Knudsen KE and Sette C: Alternative splicing of the cyclin D1 proto-oncogene is regulated by the RNA-binding protein Sam68. Cancer Res 70: 229-239, 2010.

13. Kim CJ, Nishi K, Isono T, Okuyama Y, Tambe Y, Okada Y and Inoue $\mathrm{H}$ : Cyclin D1b variant promotes cell invasiveness independent of binding to CDK4 in human bladder cancer cells. Mol Carcinog 48: 953-964, 2009.

14. Kim CJ, Tambe Y, Mukaisho K, Sugihara H, Isono T, Sonoda H, Shimizu T, Kondoh G and Inoue H: Female-specific rectal carcinogenesis in cyclin D1b transgenic mice. Carcinogenesis 35: 227-236, 2014.

15. Kim CJ, Tambe Y, Mukaisho KI, Sugihara H, Kawauchi A and Inoue $\mathrm{H}$ : Akt-dependent activation of Erk by cyclin D1b contributes to cell invasiveness and tumorigenicity. Oncol Lett 12: 4850-4856, 2016.

16. Kim CJ, Yuasa T, Kushima R, Tomoyoshi T and Seto A: Antitumor killer lymphocytes in the peripheral blood of a patient with transitional cell carcinoma of the bladder. Int J Urol 5: 230-236, 1998.

17. Tambe Y, Yoshioka-Yamashita A, Mukaisho K, Haraguchi S, Chano T, Isono T, Kawai T, Suzuki Y, Kushima R, Hattori T, et al: Tumor prone phenotype of mice deficient in a novel apoptosis-inducing gene, drs. Carcinogenesis 28: 777-784, 2007.

18. Weiswald LB, Bellet D and Dangles-Marie V: Spherical cancer models in tumor biology. Neoplasia 17: 1-15, 2015.

19. Robertson FM, Ogasawara MA, Ye Z, Chu K, Pickei R, Debeb BG, Woodward WA, Hittelman WN, Cristofanilli M and Barsky SH: Imaging and analysis of 3D tumor spheroids enriched for a cancer stem cell phenotype. J Biomol Screen 15: 820-829, 2010

20. Xu H, Tian Y, Yuan X, Wu H, Liu Q, Pestell RG and Wu K: The role of CD44 in epithelial-mesenchymal transition and cancer development. Onco Targets Ther 8: 3783-3792, 2015.

21. Cairns P, Proctor AJ and Knowles MA: Loss of heterozygosity at the RB locus is frequent and correlates with muscle invasion in bladder carcinoma. Oncogene 6: 2305-2309, 1991.

22. Cordon-Cardo C, Wartinger D, Petrylak D, Dalbagni G, Fair WR, Fuks Z and Reuter VE: Altered expression of the retinoblastoma gene product: Prognostic indicator in bladder cancer. J Natl Cancer Inst 84: 1251-1256, 1992.

23. Logothetis CJ, Xu HJ, Ro JY, Hu SX, Sahin A, Ordonez N and Benedict WF: Altered expression of retinoblastoma protein and known prognostic variables in locally advanced bladder cancer. $\mathrm{J}$ Natl Cancer Inst 84: 1256-1261, 1992.

24. Burkhart DL and Sage J: Cellular mechanisms of tumour suppression by the retinoblastoma gene. Nat Rev Cancer 8: 671-682, 2008.

25. Knudson ES and Knudson KE: Tailoring to RB: Tumor suppressor status and therapeutic response. Natl Rev 8: 714-724, 2008. 
26. Thangavel C, Boopathi E, Liu Y, Haber A, Ertel A, Bhardwaj A, Addya S, Williams N, Ciment SJ, Cotzia P, et al: RB loss promotes prostate cancer metastasis. Cancer Res 77: 982-995, 2017.

27. Wei M, Zhu L, Li Y, Chen W, Han B, Wang Z, He J, Yao H, Yang Z, Zhang Q, et al: Knocking down cyclin D1b inhibits breast cancer cell growth and suppresses tumor development in a breast cancer model. Cancer Sci 102: 1537-1544, 2011.

28. Spring FA, Dalchau R, Daniels GL, Mallinson G, Judson PA, Parsons SF, Fabre JW and Anstee DJ: The Ina and Inb blood group antigens are located on a glycoprotein of 80,000 MW (the CDw44 glycoprotein) whose expression is influenced by the In $(\mathrm{Lu})$ gene. Immunology 64: 37-43, 1988.

29. Misra S, Heldin P, Hascall VC, Karamanos NK, Skandalis SS, Markwald RR and Ghatak S: Hyaluronan-CD44 interactions as potential targets for cancer therapy. FEBS J 278: 1429-1443, 2011.

30. Ponta H, Sherman L and Herrlich PA: CD44: From adhesion molecules to signalling regulators. Nat Rev Mol Cell Biol 4 33-45, 2003

31. Turley EA, Noble PW and Bourguignon LYW: Signaling properties of hyaluronan receptors. J Biol Chem 277: 4589-4592, 2002

32. Misra S, Hascall VC, De Giovanni C, Markwald RR and Ghatak S: Delivery of CD44 shRNA/nanoparticles within cancer cells: Perturbation of hyaluronan/CD44v6 interactions and reduction in adenoma growth in Apc Min/+ MICE. J Biol Chem 284: 12432-12446, 2009.

33. Thiery JP: Epithelial-mesenchymal transitions in tumour progression. Nat Rev Cancer 2: 442-454, 2002.

34. Polyak K and Weinberg RA: Transitions between epithelial and mesenchymal states: Acquisition of malignant and stem cell traits. Nat Rev Cancer 9: 265-273, 2009.

35. Kim JB, Islam S, Kim YJ, Prudoff RS, Sass KM, Wheelock MJ and Johnson KR: N-Cadherin extracellular repeat 4 mediates epithelial to mesenchymal transition and increased motility. J Cell Biol 151: 1193-1206, 2000.
36. Lascombe I, Clairotte A, Fauconnet S, Bernardini S, Wallerand $\mathrm{H}$, Kantelip B and Bittard H: N-cadherin as a novel prognostic marker of progression in superficial urothelial tumors. Clin Cancer Res 12: 2780-2787, 2006.

37. Hazan RB, Phillips GR, Qiao RF, Norton L and Aaronson SA: Exogenous expression of $\mathrm{N}$-cadherin in breast cancer cells induces cell migration, invasion, and metastasis. J Cell Biol 148: 779-790, 2000

38. Gravdal K, Halvorsen OJ, Haukaas SA and Akslen LA: A switch from $\mathrm{E}$-cadherin to $\mathrm{N}$-cadherin expression indicates epithelial to mesenchymal transition and is of strong and independent importance for the progress of prostate cancer. Clin Cancer Res 13: 7003-7011, 2007.

39. Bryan RT: Cell adhesion and urothelial bladder cancer: The role of cadherin switching and related phenomena. Philos Trans R Soc Lond B Biol Sci 370: 20140042, 2015.

40. Jäger T, Becker M, Eisenhardt A, Tilki D, Tötsch M, Schmid KW, Romics I, Rübben H, Ergün S and Szarvas T: The prognostic value of cadherin switch in bladder cancer. Oncol Rep 23: 1125-1132, 2010.

41. Gaglione M and Messere A: Recent progress in chemically modified siRNAs. Mini Rev Med Chem 10: 578-595, 2010.

42. Herr HW, Laudone VP and Whitmore WF Jr: An overview of intravesical therapy for superficial bladder tumors. J Urol 138: 1363-1368, 1987.

43. Okamura K, Ono Y, Kinukawa T, Matsuura O, Yamada S, Ando T, Fukatsu T, Ohno Y and Ohshima S; Nagoya University Urological Oncology Group: Randomized study of single early instillation of (2"R)-4'-O-tetrahydropyranyl-doxorubicin for a single superficial bladder carcinoma. Cancer 94: 2363-2368, 2002 .

44. Nogawa M, Yuasa T, Kimura S, Tanaka M, Kuroda J, Sato K, Yokota A, Segawa H, Toda Y, Kageyama S, et al: Intravesical administration of small interfering RNA targeting PLK-1 successfully prevents the growth of bladder cancer. J Clin Invest 115: 978-985, 2005. 